\title{
Termination for the Direct Sum of Left-Linear Term Rewriting Systems - Preliminary Draft*-
}

\author{
Yoshihito Toyama \\ NTT Basic Research Laboratories \\ 3-9-11 Midori-cho, Musashino-shi, Tokyo 180, Japan \\ email: toyama\%ntt-20.ntt.jp@relay.cs.net \\ Jan Willem Klop \\ Centre for Mathematics and Computer Science \\ Kruislaan 413, 1098 SJ, Amsterdam, The Netherlands \\ Hendrik Pieter Barendregt \\ Informatica, Nijmegen University \\ Toernooiveld 1, $6525 \mathrm{ED}$, Nijmegen, The Netherlands
}

\begin{abstract}
The direct sum of two term rewriting systems is the union of systems having disjoint sets of function symbols. It is shown that two term rewriting systems both are left-linear and complete if and only if the direct sum of these systems is so.
\end{abstract}

\section{Introduction}

An important concern in building algebraic specifications is their hierarchical or modular structure. The same holds for term rewriting systems [1] which can be viewed as implementations of equational algebraic specifications. Specifically, it is of obvious interest to determine which

*This paper is an abbreviated version of the IEICE technical report COMP88-90, July 1988. Now we are preparing a final version for submission based on this draft. 
properties of term rewriting systems have a modular character, where we call a property modular if its validity for a term rewriting system, hierarchically composed of some smaller term rewriting systems, can be inferred from the validity of that property for the constituent term rewriting systems. Naturally, the first step in such an investigation considers the most basic properties of term rewriting systems: confluence, termination, unique normal form property, and similar fundamental properties as well as combinations thereof.

As to the modular structure of term rewriting systems, it is again natural to consider as a start the most simple way that term rewriting systems can be combined to form a larger term rewriting system: namely, as a disjoint sum. This means that the alphabets of the term rewriting systems to be combined are disjoint, and that the rewriting rules of the sum term rewriting system are the rules of the summand term rewriting systems together. (Without the disjointness requirement the situation is even more complicated - see for some results in this direction: Dershowitz [2], Toyama [10].) A disjoint union of two term rewriting systems $R_{0}$ and $R_{1}$ is called in our paper a direct sum, notation $R_{0} \oplus R_{1}$.

Another simplifying assumption that we will make, is that $R_{0}, R_{1}$ are homogeneous term rewriting systems, i.e. their signature is one-sorted (as opposed to the many sorted or heterogeneous case; for results about direct sums of heterogeneous term rewriting systems, see Ganzinger and Giegerich [3].)

The first result in this setting is due to Toyama [8], where it is proven that confiuence is a modular property. (I.e. $R_{0}$ and $R_{1}$ are confluent iff $R_{0} \oplus R_{1}$ is so. Here $\Leftarrow$ is trivial; $\Rightarrow$ is what we are interested in.) To appreciate the non-triviality of this fact, it may be contrasted with the fact that another fundamental property, termination, is not modular, as the following simple counterexample in Toyama [9] shows:

$$
\begin{aligned}
& R_{0} \quad\{F(0,1, x) \triangleright F(x, x, x) \\
& R_{1}\left\{\begin{array}{l}
g(x, y) \triangleright x \\
g(x, y) \triangleright y
\end{array}\right.
\end{aligned}
$$

It is trivial that $R_{0}$ and $R_{1}$ are terminating. However, $R_{0} \oplus R_{1}$ is not terminating, because $R_{0} \oplus R_{1}$ has the infinite reduction sequence:

$$
\begin{aligned}
& F(g(0,1), g(0,1), g(0,1)) \rightarrow F(0, g(0,1), g(0,1)) \rightarrow F(0,1, g(0,1)) \\
& \rightarrow F(g(0,1), g(0,1), g(0,1)) \rightarrow \cdots
\end{aligned}
$$

The above counterexample uses a non-confluent term rewriting system $R_{1}$. A more complicated counterexample to the modularity of termination, involving only confluent term rewriting 
systems, was given by Klop an Barendregt [4] (for ground terms only; for some improved versions, holding for open terms as well, and even using term rewriting systems which are irreducible, see Toyama [9]). This means that the important property of completeness of term rewriting systems (a term rewriting system is complete iff it is both confluent and terminating) is not modular, i.e. there are complete term rewriting systems $R_{0}, R_{1}$ such that $R_{0} \oplus R_{1}$ is not complete (in fact, not terminating; confluence of $R_{0} \oplus R_{1}$ is ensured by the theorem in Toyama [8]). This counterexample, however, uses non-left-linear term rewriting systems.

The point of the present paper is that left-linearity is essential; if we restrict ourselves to left-linear term rewriting systems, then completeness is modular. Thus we prove: If $R_{0}$ and $R_{1}$ are left-linear (meaning that the rewriting rules have no repeated variables in their left-handsides), then $R_{0}$ and $R_{1}$ are complete iff $R_{0} \oplus R_{1}$ is so. As left-linearity is a property which is so easily checked, and many equational algebraic specifications can be given by term rewriting systems which are left-linear, we feel that this result is worth while.

The proof, however, is rather intricate and not easily digested. A crucial element in the proof, and in general in the way that the summand term rewriting systems interact, is how terms may collapse to a subterm. The problem is that this collapsing behavior may exhibit a nondeterministic feature, which is caused by ambiguities among the rewriting rules. We hope that the present paper is of value not only because it establishes a result that in itself is simple enough, but also because of the analysis necessary for the proof which gives a kind of structure theory for disjoint combinations of term rewriting systems and which may be of relevance in other, similar, studies.

Regarding the question of modular properties in the present simple set-up, we mention the recent results by Rusinowitch [7] and Middeldorp [5]; these papers, together, contain a complete analysis of the cases in which termination for $R_{0} \oplus R_{1}$ may be concluded from termination of $R_{0}$, $R_{1}$, depending on the distribution among $R_{0}, R_{1}$ of so-called collapsing and duplicating rules.

Another useful fact is established in Middeldorp [6], where it is proven that the unique normal form property is a modular property.

\section{Notations and Definitions}

Assuming that the reader is familiar with the basic concepts and notations concerning term rewriting systems in $[1,8]$, we briefly explain notations and definitions for the following discussions.

Let $F$ be a set of function symbols, and let $V$ be a set of variable symbols. By $T(F, V)$, we denote the set of terms constructed from $F$ and $V$.

A term rewriting system $R$ is a set of rewriting rules $M \triangleright N$, where $M$ and $N$ are terms 
disjoint function symbols [8].

In this paper, we assume that two disjoint systems $R_{0}$ on $T\left(F_{0}, V\right)$ and $R_{1}$ on $T\left(F_{1}, V\right)$ both are left-linear and complete. Then we shall prove that the direct sum system $R_{0} \oplus R_{1}$ on $T\left(F_{0} \cup F_{1}, V\right)$ is terminating. From here on the notation $\rightarrow$ represents the reduction relation on $R_{0} \oplus R_{1}$.

Lemma 2.1. $R_{0} \oplus R_{1}$ is weakly normalizing, i.e., every term $M$ has a normal form (denoted by $M \downarrow)$.

Proof. Since $R_{0}$ and $R_{1}$ are terminating, $M$ can be reduced into $M \downarrow$ through innermost reduction.

The identity of terms of $T\left(F_{0} \cup F_{1}, V\right)$ (or syntactical equality) is denoted by $\equiv . \stackrel{*}{\rightarrow}$ is the transitive reflexive closure of $\rightarrow, \pm$ is the transitive closure of $\rightarrow, \equiv$ is the reflexive closure of $\rightarrow$, and $=$ is the equivalence relation generated by $\rightarrow$ (i.e., the transitive reflexive symmetric closure of $\rightarrow) . \stackrel{m}{\rightarrow}$ denotes a reduction of $m(m \geq 0)$ steps.

Definition. A root is a mapping from $T\left(F_{0} \cup F_{1}, V\right)$ to $F_{0} \cup F_{1} \cup V$ as follows: For $M \in$ $T\left(F_{0} \cup F_{1}, V\right)$,

$$
\operatorname{root}(M)= \begin{cases}f & \text { if } M \equiv f\left(M_{1}, \ldots, M_{n}\right), \\ M & \text { if } M \text { is a constant or a variable. }\end{cases}
$$

Definition. Let $M \equiv C\left[B_{1}, \ldots, B_{n}\right] \in T\left(F_{0} \cup F_{1}, V\right)$ and $C \not \equiv$. Then write $M \equiv$ $C \llbracket B_{1}, \ldots, B_{n} \rrbracket$ if $C\left[, \ldots, \rrbracket\right.$ is a context on $F_{d}$ and $\forall i, \operatorname{root}\left(B_{i}\right) \in F_{\bar{d}}(d \in\{0,1\}$ and $\bar{d}=1-d)$. Then the set $S(M)$ of the special subterms of $M$ is inductively defined as follows:

$$
S(M)= \begin{cases}\{M\} & \text { if } M \in T\left(F_{d}, V\right)(d=0 \text { or } 1), \\ \bigcup_{i} S\left(B_{i}\right) \cup\{M\} & \text { if } M \equiv C \llbracket B_{1}, \ldots, B_{n} \rrbracket(n>0) .\end{cases}
$$

The set of the special subterms having the root symbol in $F_{d}$ is denoted by $S_{d}(M)=\{N \mid N \in$ $S(M)$ and $\left.\operatorname{root}(N) \in F_{d}\right\}$.

Let $M \equiv C \llbracket B_{1}, \ldots, B_{n} \rrbracket$ and $M \stackrel{A}{\rightarrow} N$ (i.e., $N$ results from $M$ by contracting the redex occurrence $A$ ). If the redex occurrence $A$ occurs in some $B_{j}$, then we write $M \rightarrow N$; otherwise $M \underset{0}{\rightarrow} N$. Here, $\underset{i}{\rightarrow}$ and $\underset{0}{\rightarrow}$ are called an inner and an outer reduction, respectively.

Definition. For a term $M \in T\left(F_{0} \cup F_{1}, V\right)$, the rank of layers of contexts on $F_{0}$ and $F_{1}$ in $M$ is inductively defined as follows:

$$
\operatorname{rank}(M)= \begin{cases}1 & \text { if } M \in T\left(F_{d}, V\right)(d=0 \text { or } 1), \\ \max _{i}\left\{\operatorname{rank}\left(B_{i}\right)\right\}+1 & \text { if } M \equiv C \llbracket B_{1}, \ldots, B_{n} \rrbracket(n>0) .\end{cases}
$$


Lemma 2.2. If $M \rightarrow N$ then $\operatorname{rank}(M) \geq \operatorname{rank}(N)$.

Proof. It is easily obtained from the definitions of the direct sum.

Lemma 2.3. Let $M \stackrel{*}{\rightarrow} N$ and $\operatorname{root}(M), \operatorname{root}(N) \in F_{d}$. Then there exists a reduction $M \equiv M_{0} \rightarrow M_{1} \rightarrow M_{2} \rightarrow \cdots \rightarrow M_{n} \equiv N \quad(n \geq 0)$ such that $\operatorname{root}\left(M_{i}\right) \in F_{d}$ for any $i$.

Proof. Let $M \stackrel{k}{\rightarrow} N \quad(k \geq 0)$. We will prove the lemma by induction on $k$. The case $k=0$ is trivial. Let $M \rightarrow M^{\prime} \stackrel{k-1}{\rightarrow} N(k>0)$. If $\operatorname{root}\left(M^{\prime}\right) \in F_{d}$ then the lemma holds by the induction hypothesis. If $\operatorname{root}\left(M^{\prime}\right) \in F_{\bar{d}}$ then there exists a context $C$ [] with root $\in F_{d}$ such that $M \equiv C\left[M^{\prime}\right]$ and $C[] \rightarrow \square$. Thus, we can obtain a reduction $M \equiv C\left[M^{\prime}\right] \stackrel{*}{\rightarrow} C[N] \rightarrow N$ in which all terms have root symbols in $F_{d}$.

The set of terms in the reduction graph of $M$ is denoted by $G(M)=\{N \mid M \stackrel{*}{\rightarrow} N\}$. The set of terms having the root symbol in $F_{d}$ is denoted by $G_{d}(M)=\left\{N \mid N \in G(M)\right.$ and $\left.\operatorname{root}(N) \in F_{d}\right\}$.

Definition. A term $M$ is erasable iff $M \stackrel{*}{\rightarrow} x$ for some $x \in V$.

From now on we assume that every term $M \in T\left(F_{0} \cup F_{1}, V\right)$ has only $x$ as variable occurrences, unless it is stated otherwise. Since $R_{0} \oplus R_{1}$ is left-linear, this variable convention may be assumed in the following discussions without loss of generality. If we need fresh variable symbols not in terms, we use $z, z_{1}, z_{2}, \cdots$.

\section{Essential Subterms}

In this section we introduce the concept of the essential subterms. We first prove the following property:

$$
\forall N \in G_{d}(M) \exists P \in S_{d}(M), M \stackrel{*}{\rightarrow} P \stackrel{*}{\rightarrow} N
$$

Lemma 3.1. Let $M \rightarrow N$ and $Q \in S_{d}(N)$. Then, there exists some $P \in S_{d}(M)$ such that $P \equiv Q$

Proof. We will prove the lemma by induction on $\operatorname{rank}(M)$. The case $\operatorname{rank}(M)=1$ is trivial. Assume the lemma for $\operatorname{ran} k(M)<k(k>1)$, then we will show the case $\operatorname{ran} k(M)=k$. Let $M \equiv C \llbracket M_{1}, \ldots, M_{n} \rrbracket(n>0)$ and $M \stackrel{A}{\rightarrow} N$.

Case 1. $M \equiv C \llbracket M_{1}, \ldots, M_{r}, \ldots, M_{n} \rrbracket \underset{0}{\stackrel{A}{\rightarrow}} N \equiv M_{r}$.

Then $S_{d}(N) \subseteq S_{d}(M)$. 
Case 2. $M \equiv C \llbracket M_{1}, \ldots, M_{n} \rrbracket \underset{\circ}{\stackrel{A}{\rightarrow}} N \equiv C^{\prime} \llbracket M_{i_{1}}, \ldots, M_{i_{p}} \rrbracket\left(1 \leq i_{j} \leq n\right)$.

If $\operatorname{root}(M) \in F_{d}$ then

$S_{d}(M)=\{M\} \cup \cup_{i} S_{d}\left(M_{i}\right)$,

$S_{d}(N)=\{N\} \cup \cup_{j} S_{d}\left(M_{i_{j}}\right)$.

Thus the lemma holds since $\bigcup_{j} S_{d}\left(M_{i_{j}}\right) \subseteq \bigcup_{i} S_{d}\left(M_{i}\right)$, and $M \rightarrow N$.

If $\operatorname{root}(M) \in F_{\bar{d}}$ then $S_{d}(N)=\cup_{j} S_{d}\left(M_{i_{j}}\right) \subseteq \bigcup_{i} S_{d}\left(M_{i}\right)=S_{d}(M)$.

Case 9. $M \equiv C \llbracket M_{1}, \ldots, M_{r}, \ldots, M_{n} \rrbracket \underset{i}{\stackrel{A}{\rightarrow}} N \equiv C\left[M_{1}, \ldots, M_{r}^{\prime}, \ldots, M_{n}\right]$ where $M_{r} \stackrel{A}{\rightarrow} M_{r}^{\prime}$. If $\operatorname{root}(M) \in F_{d}$ then

$S_{d}(M)=\{M\} \cup S_{d}\left(M_{r}\right) \cup \bigcup_{i \neq r} S_{d}\left(M_{i}\right)$,

$S_{d}(N) \subseteq\{N\} \cup S_{d}\left(M_{r}^{\prime}\right) \cup \cup_{i \neq r} S_{d}\left(M_{i}\right)$.

If $\operatorname{root}(M) \in F_{\bar{d}}$ then

$S_{d}(M)=S_{d}\left(M_{r}\right) \cup \bigcup_{i \neq r} S_{d}\left(M_{i}\right)$,

$S_{d}(N)=S_{d}\left(M_{r}^{\prime}\right) \cup \bigcup_{i \neq r} S_{d}\left(M_{i}\right)$

By the induction hypothesis, $\forall Q \in S_{d}\left(M_{r}^{\prime}\right) \exists P \in S_{d}\left(M_{r}\right), P \equiv Q$ for the both $\operatorname{root}(M) \in F_{d}$ and $\operatorname{root}(M) \in F_{\bar{d}}$. Thus the lemma holds.

$R_{e}$ consists of the single rule $e(x) \triangleright x . \vec{e}$ denotes the reduction relation of $R_{e}$, and $\overrightarrow{e^{\prime}}$ denotes the reduction relation of $R_{e} \oplus\left(R_{0} \oplus R_{1}\right)$ such that if $C[e(P)] \underset{e^{\prime}}{\stackrel{\Delta}{\Rightarrow}} N$ then the redex occurrence $\Delta$ does not occur in $P$. It is easy to show the confluence property of $\overrightarrow{e^{\prime}}$.

From here on, $C\left[e\left(P_{1}\right), \cdots, e\left(P_{p}\right)\right]$ denotes a term such that $C\left[P_{1}, \cdots, P_{p}\right] \in T\left(F_{0} \cup F_{1}, V\right)$, i.e., $C$ and $P_{i}$ contain no $e$.

Lemma 3.2. Let $C\left[e\left(P_{1}\right), \cdots, e\left(P_{i-1}\right), e\left(P_{i}\right), e\left(P_{i+1}\right), \cdots, e\left(P_{p}\right)\right] \frac{k}{e^{\prime}} e\left(P_{i}\right)$. Then $C\left[P_{1}, \cdots\right.$, $\left.P_{i-1}, e\left(P_{i}\right), P_{i+1}, \cdots, P_{p}\right] \underset{e^{\prime}}{\frac{k^{\prime}}{\longrightarrow}} e\left(P_{i}\right)\left(k^{\prime} \leq k\right)$.

Proof. It is easily obtained from the definition and the left-linearity of the reduction $\overrightarrow{e^{\prime}}$.

Let $M \equiv C[P] \in T\left(F_{0} \cup F_{1}, V\right)$ be a term containing no function symbol $e$. Now, consider $C[e(P)]$ by replacing the occurrence $P$ in $M$ with $e(P)$. Assume $C[e(P)] \underset{e^{\prime}}{\stackrel{*}{*} e}(P)$. Then, by tracing the reduction path, we can also obtain the reduction $M \equiv C[P] \stackrel{*}{\rightarrow} P$ (denoted by $M \underset{\text { pull }}{\stackrel{*}{\longrightarrow}} P$ ) under $R_{0} \oplus R_{1}$. We say that the reduction $M \underset{\text { pull }}{\stackrel{*}{\longrightarrow}} P$ pulls up the occurrence $P$ from $M$. 
Example 3.1. Consider the two systems $R_{0}$ and $R_{1}$ :

$$
\begin{aligned}
& R_{0} \quad\left\{\begin{array}{l}
F(x) \rightarrow G(x, x) \\
G(C, x) \rightarrow x
\end{array}\right. \\
& R_{1} \quad\{h(x) \rightarrow x
\end{aligned}
$$

Then we have the reduction:

$F(e(h(C))) \underset{e^{\prime}}{\rightarrow} G\left(e(h(C), e(h(C))) \underset{e^{\prime}}{\rightarrow} G(h(C), e(h(C))) \underset{e^{\prime}}{\rightarrow} G(C, e(h(C))) \underset{e^{\prime}}{\rightarrow} e(h(C))\right.$.

Hence $F(h(C)) \underset{\text { pull }}{\stackrel{*}{\rightarrow}} h(C)$. However, we cannot obtain $F(z) \underset{\text { pull }}{\stackrel{*}{\longrightarrow}}$. Thus, in general, we cannot obtain $C[z] \underset{\text { pull }}{\stackrel{*}{\longrightarrow}} z$ from $C[P] \underset{p u l l}{\stackrel{*}{\longrightarrow}} P$. $\square$

Lemma 3.3. Let $P \stackrel{*}{\rightarrow} Q$ and let $C[Q] \underset{\text { pull }}{\stackrel{*}{\rightarrow}} Q$. Then $C[P] \underset{\text { pull }}{\stackrel{*}{\rightarrow}} P$.

Proof. Let $M \equiv C[e(Q)] \stackrel{k}{e^{\prime}} e(Q)$. We will prove the lemma by induction on $k$. The case $k=0$ is trivial. Let $M \equiv C[e(Q)] \underset{e^{\prime}}{\longrightarrow} C^{\prime}[e(Q), \cdots, e(Q), \cdots, e(Q)] \underset{e^{\prime}}{\frac{k-1}{\prime}} e(Q)$. Then, from Lemma 3.2 we can obtain the following reduction:

$C^{\prime}[Q, \cdots, e(Q), \cdots, Q] \underset{\mathrm{e}^{\prime}}{\frac{k^{\prime}}{\longrightarrow}} e(Q)\left(k^{\prime} \leq k-1\right)$.

By using the induction hypothesis, $C^{\prime}[Q, \cdots, e(P), \cdots, Q] \underset{e^{\prime}}{*} e(P)$. Therefore, we can obtain $C[e(P)] \underset{e^{\prime}}{\longrightarrow} C^{\prime}[e(P), \cdots, e(P), \cdots, e(P)] \stackrel{*}{e^{\prime}} C^{\prime}[Q, \cdots, e(P), \cdots, Q] \stackrel{*}{\underset{e^{\prime}}{\longrightarrow}} e(P)$ from $P \stackrel{*}{\rightarrow} Q$.

Lemma 3.4. $\forall N \in G_{d}(M) \exists P \in S_{d}(M), M \stackrel{*}{\stackrel{*}{\rightarrow u l l}} P \stackrel{*}{\rightarrow} N$.

Proof. If $\operatorname{root}(M) \in F_{d}$ then the above property is trivial by taking $M$ as $P$. Thus we consider only the non trivial case of $\operatorname{root}(M) \in F_{\bar{d}}$. Let $M \stackrel{k}{\longrightarrow} N$. We will prove the lemma by induction on $k$. The case $k=1$ is trivial since $M \equiv C \llbracket M_{1}, \ldots, M_{r}, \ldots, M_{n} \rrbracket \rightarrow N \equiv M_{r}$ for some $r$ (i.e., take $P \equiv M_{r}$ ). Assume the lemma for $k-1$. We will prove the case $k$. Let $M \rightarrow M^{\prime} \stackrel{k-1}{\longrightarrow} N$.

Case 1. $\operatorname{root}\left(M^{\prime}\right) \in F_{d}$.

Then $M \equiv C \llbracket M_{1}, \ldots, M_{r}, \ldots, M_{n} \rrbracket \rightarrow M^{\prime} \equiv M_{r}$ for some $r$. Take $P \equiv M_{r}$.

Case 2. $\operatorname{root}\left(M^{\prime}\right) \in F_{\bar{d}}$. 
By using the induction hypothesis, $\exists P^{\prime} \in S_{d}\left(M^{\prime}\right), M^{\prime} \underset{\text { pull }}{\stackrel{*}{\rightarrow}} P^{\prime} \stackrel{*}{\rightarrow} N$. Here, from Lemma 3.1, there exists some $P \in S_{d}(M)$ such that $P \equiv P^{\prime}$. We will consider the following two subcases:

Case 2.1. $P \rightarrow P^{\prime}$. Then $M \equiv C[P] \rightarrow M^{\prime} \equiv C\left[P^{\prime}\right]$. Thus, by using Lemma $3.3, M \equiv$ $C[P] \underset{\text { pull }}{\stackrel{*}{\rightarrow}} P \rightarrow P^{\prime} \stackrel{*}{\rightarrow} N$.

Case 2.2. $P \equiv P^{\prime}$. Then, for some context $C^{\prime}\{, \cdots],, M \equiv C[P] \rightarrow M^{\prime} \equiv C^{\prime}[P, \cdots, P, \cdots, P]$ and $C^{\prime}[P, \cdots, e(P), \cdots, P] \stackrel{*}{\epsilon^{\prime}} e(P)$. Therefore

$C[e(P)] \rightarrow C^{\prime}[e(P), \cdots, e(P), \cdots, e(P)] \stackrel{*}{e} C^{\prime}[P, \cdots, e(P), \cdots, P] \stackrel{*}{e^{\prime}} e(P)$. Thus $M \equiv C[P]$ $\stackrel{*}{\rightarrow} P \stackrel{*}{\rightarrow} N$.

Now, we introduce the concept of the essential subterms. The set $E_{d}(M)$ of the essential subterms of the term $M \in T\left(F_{0} \cup F_{1}, V\right)$ is defined as follows:

$$
E_{d}(M)=\left\{P \mid M \underset{\text { pull }}{\stackrel{*}{\rightarrow}} P \in S_{d}(M) \text { and } \neg \exists Q \in S_{d}(M)[M \underset{\text { pull }}{\stackrel{*}{\rightarrow}} Q \pm P]\right\} \text {. }
$$

The following lemmas are easily obtained from the definition of the essential subterms and Lemma 3.4.

Lemma 3.5. $\forall N \in G_{d}(M) \exists P \in E_{d}(M), P \stackrel{*}{\rightarrow} N$.

Lemma 3.6. $E_{d}(M)=\phi$ iff $G_{d}(M)=\phi$.

We say $M$ is deterministic for $d$ if $\left|E_{d}(M)\right|=1 ; M$ is nondeterministic for $d$ if $\left|E_{d}(M)\right| \geq 2$. The following lemma plays an important role in the next section.

Lemma 3.7 If $\operatorname{root}(M \downarrow) \in F_{d}$ then $\left|E_{d}(M)\right|=1$, i.e., $M$ is deterministic for $d$.

Proof. See Appendix in [11].

\section{Termination for the Direct Sum}

In this section we will show that $R_{0} \oplus R_{1}$ is terminating. Roughly speaking, termination is proven by showing that any infinite reduction $M_{0} \rightarrow M_{1} \rightarrow M_{2} \rightarrow \cdots$ of $R_{0} \oplus R_{1}$ can be translated into an infinite reduction $M_{0}^{\prime} \rightarrow M_{1}^{\prime} \rightarrow M_{2}^{\prime} \rightarrow \cdots$ of $R_{d}$.

We first define the term $M^{d} \in T\left(F_{d}, V\right)$ for any term $M$ and any $d$. 
Definition. For any $M$ and any $d, M^{d} \in T\left(F_{d}, V\right)$ is defined by induction on $\operatorname{rank}(M)$ :

(1) $M^{d} \equiv M \quad$ if $M \in T\left(F_{d}, V\right)$.

(2) $M^{d} \equiv x \quad$ if $E_{d}(M)=\phi$.

(3) $M^{d} \equiv C\left[M_{1}^{d}, \cdots, M_{m}^{d}\right] \quad$ if $\operatorname{root}(M) \in F_{d}$ and $M \equiv C \llbracket M_{1}, \cdots, M_{m} \rrbracket(m>0)$.

(4) $M^{d} \equiv P^{d} \quad$ if $\operatorname{root}(M) \in F_{d}$ and $E_{d}(M)=\{P\}$. Note that $\operatorname{rank}(P)<\operatorname{rank}(M)$.

(5) $M^{d} \equiv C_{1}\left[C_{2}\left[\cdots C_{p-1}\left[C_{p}[x]\right] \cdots\right]\right]$ if $\operatorname{root}(M) \in F_{\bar{d}}, E_{d}(M)=\left\{P_{1}, \cdots, P_{p}\right\}(p>1)$, and every $P_{i}^{d}$ is erasable. Here $P_{i}^{d} \equiv C_{i}[x] \underset{\text { pull }}{\stackrel{*}{\longrightarrow}} x \quad(i=1, \cdots, p)$. Note that, for any $i, \operatorname{rank}\left(P_{i}\right)<$ $\operatorname{rank}(M)$ and $M^{d} \stackrel{*}{\rightarrow} P_{i}^{d}$.

(6) $M^{d} \equiv x$ if $\operatorname{root}(M) \in F_{d},\left|E_{d}(M)\right| \geq 2$, and not (5).

Note that $M^{d}$ is not unique if a subterm of $M^{d}$ is constructed with (5) in the above definition.

Lemma 4.1. $\operatorname{root}(M \downarrow) \notin F_{d}$ iff $M^{d} \downarrow \equiv x$.

Proof. Instead of the lemma, we will prove the following claim:

Claim. If $\operatorname{root}(M \downarrow) \notin F_{d}$ then $M^{d} \downarrow \equiv x$. If $\operatorname{root}(M \downarrow) \in F_{d}$ and $M \downarrow \equiv \hat{C} \llbracket M_{1}, \cdots, M_{m} \rrbracket$ then $M^{d} \downarrow \equiv \hat{C}[x, \cdots, x]$.

Proof of the Claim. We will prove the lemma by induction on $\operatorname{rank}(M)$. The case $\operatorname{rank}(M)=$ 1 is trivial by the definition of $M^{d}$. Assume the lemma for $\operatorname{rank}(M)<k(k \geq 2)$. Then we will prove the case $\operatorname{ran} k(M)=k$.

Case 1. $\operatorname{root}(M) \in F_{d}$.

Let $M \equiv C \llbracket M_{1}, \cdots, M_{m} \rrbracket$. Then $M^{d} \equiv C\left[M_{1}^{d}, \cdots, M_{m}^{d}\right]$. We may assume that $\operatorname{root}\left(M_{i} \downarrow\right.$ )$\notin F_{d} \quad(1 \leq i<p)$ and $\operatorname{root}\left(M_{j} \downarrow\right) \in F_{d} \quad(p \leq j \leq m)$ without loss of generality. Let $M_{j} \downarrow \equiv \hat{C}_{j} \llbracket N_{j, 1}, \cdots, N_{j, n} \rrbracket \quad(p \leq j \leq m)$. Then, by using the induction hypothesis, $M_{i}^{d} \downarrow \equiv x$ $(1 \leq i<p)$ and $M_{j}^{d} \downarrow \equiv \hat{C}_{j}[x, \cdots, x] \quad(p \leq j \leq m)$. Thus $M \downarrow \equiv C\left[M_{1} \downarrow, \cdots, M_{m} \downarrow\right) \downarrow$

$\equiv C\left[M_{1} \downarrow, \cdots, M_{p-1} \downarrow, \hat{C}_{p} \llbracket N_{p, 1}, \cdots, N_{p, n_{p}} \rrbracket, \cdots, \hat{C_{m}} \llbracket N_{m, 1}, \cdots, N_{m, n_{m}} \rrbracket\right]$ and $M^{d} \downarrow \equiv C\left[M_{1}^{d} \downarrow, \cdots, M_{m}^{d} \downarrow\right] \downarrow \equiv C\left[x, \cdots, x, \hat{C_{p}}[x, \cdots, x], \cdots, \hat{C_{m}}[x, \cdots, x]\right] \downarrow$. Note that $M_{i} \downarrow$ $(1 \leq i<p), N_{p, 1}, \cdots, N_{m, n_{m}}$ are normal forms having root symbols not in $F_{d}$. Therefore, if $\operatorname{root}(M \downarrow) \notin F_{d}$ then $C\left[x, \cdots, x, \hat{C_{p}}[x, \cdots, x], \cdots, \hat{C_{m}}[x, \cdots, x]\right] \downarrow \equiv x ;$ if $\operatorname{root}(M \downarrow) \in F_{d}$ then we have a context

$\hat{C}[, \cdots,] \equiv C\left[, \cdots, \hat{C}_{p}\lfloor, \cdots],, \cdots, \hat{C_{m}} \llbracket, \cdots,\right] \downarrow \downarrow$ such that $M \downarrow \equiv \hat{C} \llbracket N_{1}, \cdots, N_{n} \rrbracket$ where $N_{i} \in\left\{M_{1} \downarrow, \cdots, M_{p-1} \downarrow, N_{p, 1}, \cdots, N_{m_{,}, n_{m}}\right\}$ and $M^{d} \downarrow \equiv \hat{C}[x, \cdots, x] \neq \equiv$. 
Case 2. $\operatorname{root}(M) \notin F_{d}$.

Consider three subcases:

Case 2.1. $E_{d}(M)=\phi$.

From Lemma 3.6, $\operatorname{root}(M \downarrow) \notin F_{d}$. Since $M^{d} \equiv x, M^{d} \downarrow \equiv x$.

Case 2.2. $E_{d}(M)=\{P\}$.

Then $M^{d} \equiv P^{d}$. Note that $\operatorname{rank}(P)<k$. Since $M \downarrow \equiv P \downarrow$ and $M^{d} \downarrow \equiv P^{d} \downarrow$, the claim follows by using the induction hypothesis.

Case 2.3. $E_{d}(M)=\left\{P_{1}, \cdots, P_{p}\right\}(p>1)$.

Note that $\operatorname{rank}\left(P_{i}\right)<k$ for any $i$. From Lemma $3.7, \operatorname{root}(M \downarrow) \notin F_{d}$. Since $M \downarrow \equiv P_{i} \downarrow$, it is clear that $\operatorname{root}\left(P_{i} \downarrow\right) \notin F_{d}$ for all $i$. Thus, we have $P_{i}^{d} \downarrow \equiv x$ by the induction hypothesis. From case (5) in the definition of $M^{d}$, it follows that $M^{d} \downarrow \equiv x$.

Note. Let $E_{d}(M)=\left\{P_{1}, \cdots, P_{p}\right\}(p>1)$. Then, from Lemma 3.7 and Lemma 4.1, it follows that every $P_{i}^{d}$ is erasable. Hence case (6) in the definition of $M^{d}$ can be removed.

Lemma 4.2. If $P \in E_{d}(M)$ then $M^{d} \stackrel{*}{\rightarrow} P^{d}$.

Proof. Obvious from the definition of $M^{d}$ and the above note.

We wish to translate directly an infinite reduction $M_{0} \rightarrow M_{1} \rightarrow M_{2} \rightarrow \cdots$ into an infinite reduction $M_{0}^{d} \stackrel{*}{\rightarrow} M_{1}^{d} \stackrel{*}{\rightarrow} M_{2}^{d} \stackrel{*}{\rightarrow} \cdots$. However, the following example shows that $M_{i} \rightarrow M_{i+1}$ cannot be translated into $M_{i}^{d} \stackrel{*}{\rightarrow} M_{i+1}^{d}$ in general.

Example 4.1. Consider the two systems $R_{0}$ and $R_{1}$ :

$$
\begin{aligned}
& R_{0}\left\{\begin{array}{l}
F(C, x) \rightarrow x \\
F(x, C) \rightarrow x
\end{array}\right. \\
& R_{1}\left\{\begin{array}{l}
f(x) \rightarrow g(x) \\
f(x) \rightarrow h(x) \\
g(x) \rightarrow x \\
h(x) \rightarrow x
\end{array}\right.
\end{aligned}
$$

Let $M \equiv F(f(C), h(C)) \rightarrow N \equiv F(g(C), h(C))$. Then $E_{1}(M)=\{f(C)\}$ and $E_{1}(N)=$ $\{g(C), h(C)\}$. Thus $M^{1} \equiv f(x), N^{1} \equiv g(h(x))$. It is obvious that $M^{1} \stackrel{*}{\rightarrow} N^{1}$ does not hold. 
Now we will consider to translate indirectly an infinite reduction of $R_{0} \oplus R_{1}$ into an infinite reduction of $R_{d}$.

We write $M \equiv N$ when $M$ and $N$ have the same outermost-layer context, i.e., $M \equiv C \llbracket M_{1}, \cdots$, $M_{m} \rrbracket$ and $N \equiv C \llbracket N_{1}, \cdots, N_{m} \rrbracket$ for some $M_{i}, N_{i}$.

Lemma 4.3. Let $A \underset{i}{\stackrel{*}{\rightarrow}} M, M \underset{0}{\rightarrow} N, A \underset{o}{\rightarrow} M$, and $\operatorname{root}(M), \operatorname{root}(N) \in F_{d}$. Then, for any $A^{d}$ there exist $B$ and $B^{d}$ such that

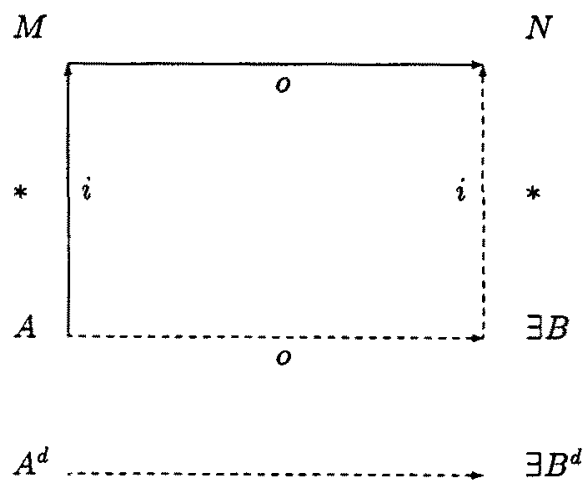

Proof. Let $A \equiv C \llbracket A_{1}, \cdots, A_{m} \rrbracket, M \equiv C \llbracket M_{1}, \cdots, M_{m} \rrbracket, N \equiv C^{\prime} \llbracket M_{i_{1}}, \cdots, M_{i_{n}} \rrbracket\left(i_{j} \in\right.$ $\{1, \cdots, m\})$. Take $B \equiv C^{\prime} \llbracket A_{i_{1}}, \cdots, A_{i_{n}} \rrbracket$. Then, we can obtain $A \underset{\circ}{\rightarrow} B$ and $B \underset{i}{*} N$. From $A^{d} \equiv C\left[A_{1}^{d}, \cdots, A_{m}^{d}\right]$ and $B^{d} \equiv C^{\prime}\left[A_{i_{1}}^{d}, \cdots, A_{i_{n}}^{d}\right]$, it follows that $A^{d} \rightarrow B^{d}$. $\square$

Lemma 4.4. Let $M \stackrel{*}{\rightarrow} N, \operatorname{root}(N) \in F_{d}$. Then, for any $M^{d}$ there exist $A(A \equiv N)$ and $A^{d}$ such that 


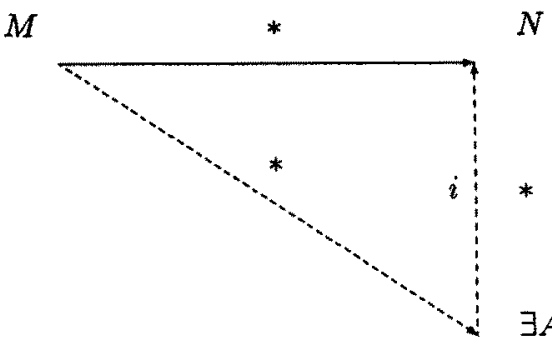

$$
N
$$

$\exists A$

$(A \equiv \overline{\bar{o}} N)$

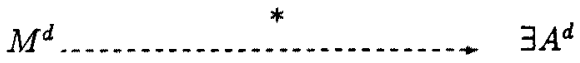

Proof. We will prove the lemma by induction on $\operatorname{rank}(M)$. The case $\operatorname{rank}(M)=1$ is trivial by taking $A \equiv N$. Assume the lemma for $\operatorname{ran} k(M)<k$. Then we will prove the case $\operatorname{rank}(M)=k$. We start from the following claim.

Claim. The lemma holds if $M \underset{i}{\stackrel{*}{\rightarrow}} N$.

Proof of the Claim. Let $M \equiv C\left[M_{1}, \cdots, M_{m} \rrbracket \stackrel{*}{\underset{i}{*}} N \equiv C\left[N_{1}, \cdots, N_{m}\right]\right.$ where $M_{i} \stackrel{*}{\rightarrow} N_{i}$ for every i. We may assume that $N_{1} \equiv x, \cdots, N_{p-1} \equiv x, \operatorname{root}\left(N_{i}\right) \in F_{d}(p \leq i \leq q-1)$, and $\operatorname{root}\left(N_{j}\right) \in$ $F_{\bar{d}}(q \leq j \leq m)$ without loss of generality. Thus $N \equiv C\left[x, \cdots, x, N_{p}, \cdots, N_{q-1}, N_{q}, \cdots, N_{m}\right]$. Then, by using the induction hypothesis, every $M_{i}(p \leq i \leq q-1)$ has $A_{i}\left(A_{i} \equiv N_{i}\right)$ and $A_{i}^{d}$ such that

$M_{\text {i }}$

$N_{i}$

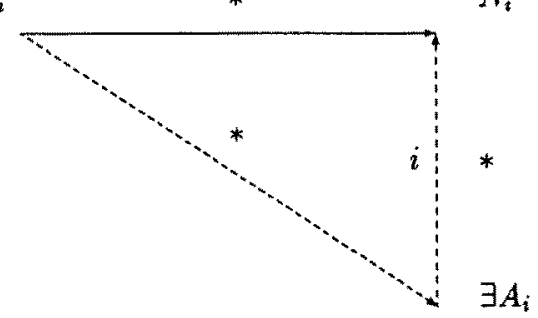

$\left(A_{i} \equiv N_{i}\right)$

$M_{i}^{d}$

$\exists A_{i}^{d}$ 
Now, take $A \equiv C\left[x, \cdots, x, A_{p}, \cdots, A_{q-1}, M_{q}, \cdots, M_{m}\right]$. It is obvious that $M \stackrel{*}{\rightarrow} A$. From Lemma 2.3, we can have the reductions $M_{j} \stackrel{*}{\rightarrow} N_{j} \quad(q \leq j \leq m)$ in which every term has a root symbol in $F_{d}$. Thus it follows that $A \underset{i}{\stackrel{*}{\rightarrow}} N$ and $A \equiv N$. From Lemma 4.1 and $M_{i} \downarrow \equiv x$ $(1 \leq i<p), M_{i}^{d} \downarrow \equiv x$. Therefore, since

$$
\begin{aligned}
& M^{d} \equiv C\left[M_{1}^{d}, \cdots, M_{p-1}^{d}, M_{p}^{d}, \cdots, M_{q-1}^{d}, M_{q}^{d}, \cdots, M_{m}^{d}\right] \\
& \text { and } A^{d} \equiv C\left[x, \cdots, x, A_{p}^{d}, \cdots, A_{q-1}^{d}, M_{q}^{d}, \cdots, M_{m}^{d}\right], \text { it follows that } M^{d} \stackrel{*}{\rightarrow} A^{d} . \text { (end of the claim) }
\end{aligned}
$$

Now we will prove the lemma for $\operatorname{rank}(M)=k$. Consider two cases.

Case 1. $\operatorname{root}(M) \in F_{d}$.

From Lemma 2.3, we may assume that every term in the reduction $M \stackrel{*}{\rightarrow} N$ has a root symbol

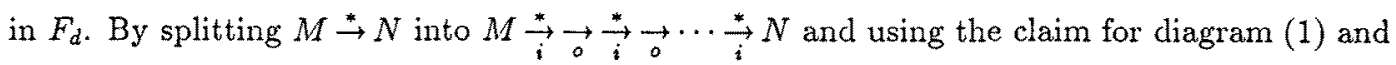
Lemma 5.1 for diagram (2), we can draw the following diagram:

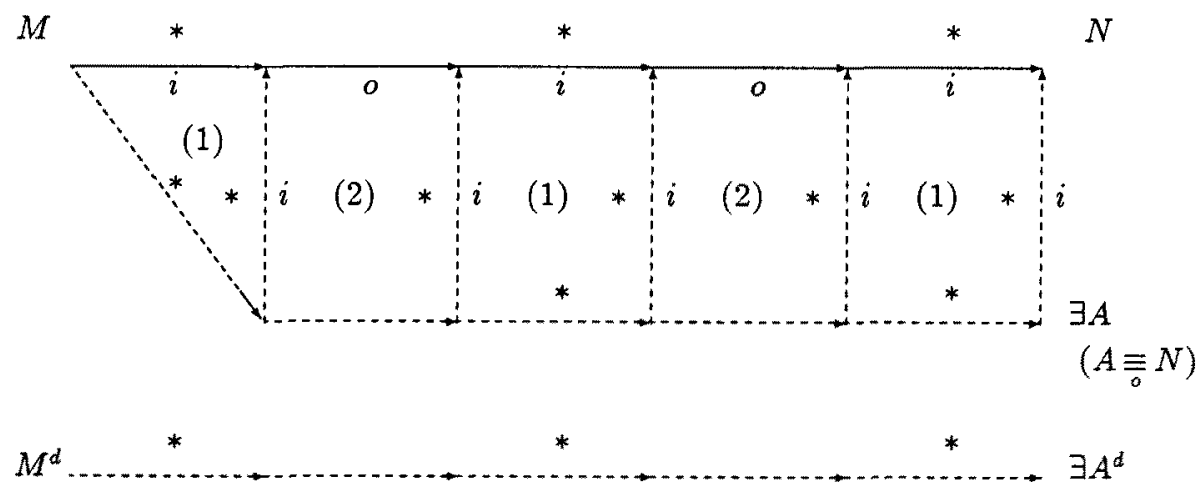

Note that if $M^{\prime} \underset{i}{\stackrel{*}{\rightarrow}} M^{\prime \prime} \underset{i}{\stackrel{*}{\rightarrow}} M^{\prime \prime \prime}$ then $M^{\prime} \underset{i}{\stackrel{*}{\rightarrow}} M^{\prime \prime \prime} ;$ thus, the claim can be applied to diagram $(1)$ in the above diagram.

Case 2. $\operatorname{root}(M) \in F_{\bar{d}}$.

Then we have some essential subterm $Q \in E_{d}(M)$ such that $M \stackrel{*}{\rightarrow} Q \stackrel{*}{\rightarrow} N$. From Lemma 4.2 , it follows that $M^{d} \stackrel{*}{\rightarrow} Q^{d}$. It is obvious that $\operatorname{ran} k(Q)<k$. Hence, we have the following diagram, where diagram (1) is obtained by the induction hypothesis: 


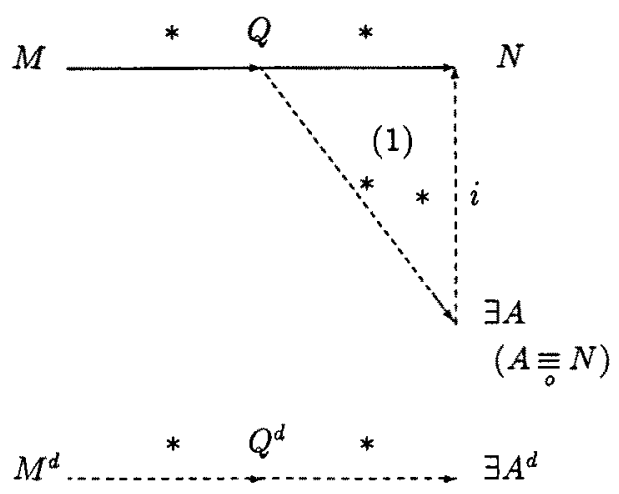

Now we can prove the following theorem:

Theorem 4.1. No term $M$ has an infinite reduction.

Proof. We will prove the theorem by induction on $\operatorname{rank}(M)$. The case $\operatorname{rank}(M)=1$ is trivial. Assume the theorem for $\operatorname{rank}(M)<k$. Then, we will show the case $\operatorname{ran} k(M)=k$. Suppose $M$ has an infinite reduction $M \rightarrow \rightarrow \rightarrow \cdots$. From the induction hypothesis, we can have no infinite inner reduction $\vec{i} \vec{i} \vec{i} \vec{i}$ in this reduction. Thus, $\overrightarrow{0}$ must infinitely appear in the infinite reduction. From the induction hypothesis, all of the terms appearing in this reduction have the same rank; hence, their root symbols are in $F_{d}$ if $\operatorname{root}(M) \in F_{d}$. Hence, by a similar construction of diagrams as for Case 1 in the proof of Lemma 4.4, it follows that $M^{d}$ has an infinite reduction. This contradicts that $R_{d}$ is terminating.

Corollary 4.1. Two term rewriting systems $R_{0}$ and $R_{1}$ are left-linear and complete iff the direct sum $R_{0} \oplus R_{1}$ is so.

Proof. $\Leftarrow$ is trivial. $\Rightarrow$ follows from Theorem 4.1 and the theorem in Toyama [8] stating that two term rewriting systems $R_{0}$ and $R_{1}$ are confluent iff the direct sum $R_{0} \oplus R_{1}$ is so.

\section{References}

[1] G. Huet and D. C. Oppen, Equations and rewrite rules: a survey, in: R. Book, ed., Formal 
languages: perspectives and open problems (Academic Press, 1980) 349-393.

[2] N. Dershowitz. Termination of linear rewriting systems: Preliminary version, Lecture Notes in Comput. Sci. 115 (Springer-Verlag, 1981) 448-458.

[3] H. Ganzinger and R. Giegerich, A note on termination in combinations of heterogeneous term rewriting systems, EATCS Bulletin 31 (1987) 22-28.

[4] J. W. Klop and H. P. Barendregt, Private communication (January 19, 1986).

[5] A. Middeldorp, A sufficient condition for the termination of the direct sum of term rewriting systems, Preliminary Draft, Report IR-150, Free University, Amsterdam (1988).

[6] A. Middeldorp, Modular aspects of properties of term rewriting systems related to normal forms, Preliminary Draft, Free University, Amsterdam (September 1988).

[7] M. Rusinowitch, On termination of the direct sum of term rewriting systems, Inform. Process. Lett. 26 (1987) 65-70.

[8] Y. Toyama, On the Church-Rosser property for the direct sum of term rewriting systems, J. ACM 34 (1987) 128-143.

[9] Y. Toyama, Counterexamples to termination for the direct sum of term rewriting systems, Inform. Process. Lett. 25 (1987) 141-143.

[10] Y. Toyama, Commutativity of term rewriting systems, in: K. Fuchi and L. Kott, eds., Programming of Future Generation Computer II (Norht Holland 1988) 393-407.

[11] Y. Toyama, J.W. Klop, H.P. Barendregt, Termination for the direct sum of left-linear term rewriting systems: Preliminary draft, IEICE technical report COM88-30 (July 1988) 47-55. 